\title{
AUGNLG: Few-shot Natural Language Generation using Self-trained Data Augmentation
}

\author{
Xinnuo Xu' ${ }^{1}$, Guoyin Wang ${ }^{2}$, Young-Bum Kim ${ }^{2}$, Sungjin Lee ${ }^{2}$ \\ ${ }^{1}$ The Interaction Lab, Heriot-Watt University, Edinburgh \\ ${ }^{2}$ Amazon Alexa AI, Seattle, WA, USA \\ xx6@hw.ac.uk, guoyiwan, youngbum, sungjinleamazon.com
}

\begin{abstract}
Natural Language Generation (NLG) is a key component in a task-oriented dialogue system, which converts the structured meaning representation (MR) to the natural language. For large-scale conversational systems, where it is common to have over hundreds of intents and thousands of slots, neither template-based approaches nor model-based approaches are scalable. Recently, neural NLGs started leveraging transfer learning and showed promising results in few-shot settings. This paper proposes AUGNLG, a novel data augmentation approach that combines a self-trained neural retrieval model with a few-shot learned NLU model, to automatically create MR-toText data from open-domain texts. The proposed system mostly outperforms the state-ofthe-art methods on the FEWSHOTWOZ data in both BLEU and Slot Error Rate. We further confirm improved results on the FEWSHOTSGD data and provide comprehensive analysis results on key components of our system. Our code and data are available at https: //github.com/XinnuoXu/AugNLG.
\end{abstract}

\section{Introduction}

Large-scale conversational systems provide a natural interface to achieve various daily-life tasks. Natural Language Generation (NLG) is a key component in such a system to convert the structured meaning representation (MR) to the natural language, as shown in Figure 1. In task-oriented dialogue systems, NLG is typically accomplished by filling out a basic set of developer-provided templates, leading to a conversational system generating unnatural, robotic responses. In order to make the system sound more human-like, model-based NLG approaches, in particular neural models, have recently been gaining an increasing traction (Gao et al., 2018; Wen et al., 2015). However, neither the template-based approaches nor the model-based

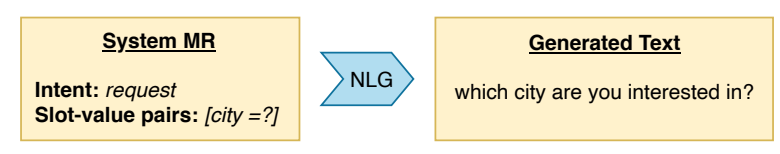

Figure 1: An example of NLG task. The model takes in the system MR, which consists of an intent with slot value pairs, and outputs text in natural language.

approaches are sufficiently scalable for large-scale conversational systems, where it is common to have over hundreds of intents and thousands of slots.

With the rise of neural transfer learning for NLP using pretrained LMs, recently, neural NLGs started to leverage transfer learning and showed some promising results (Radford et al., 2019; Brown et al., 2020; Dai et al., 2019; Edunov et al., 2019). In particular, Peng et al. (2020) proposed FEWSHOTWOZ, the first NLG benchmark test in few-shot learning settings, and achieved a SOTA performance by leveraging existing MR-to-Text data sets via task-specific continued pre-training. Despite the improved result, their approach leaves little room for further improvements as MR-to-Text data are expensive to obtain for new domains, practically circling back to the same scalability problem after exhausting the existing data.

In order to go beyond this restriction, this paper proposes AUGNLG, a novel data augmentation approach, that automatically creates MR-toText data from open-domain texts by combining a self-trained neural retrieval model with a few-shot learned NLU model. Since our data augmentation approach is orthogonal to the prior transfer learning approaches, one can use our approach in conjunction with other approaches. In experiments, we empirically show that AUGNLG mostly boosts the performance of both the fine-tuned GPT-2 (FTGPT) (Radford et al., 2019) and SC-GPT (Peng et al., 2020), the continued pretraining approach with existing MR-to-Text data, on the FEWSHOT- 


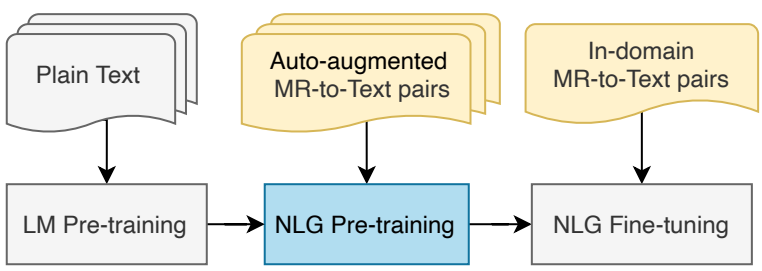

Figure 2: The training procedure for AUGNLG.

WOZ task. Furthermore, we construct another fewshot learning testbed, FEWSHOTSGD, out of the Schema-Guided Dialogue (SGD) corpus (Rastogi et al., 2020) and confirm improved results by applying AUGNLG to the FT-GPT. ${ }^{1}$ Finally, we provide comprehensive analysis results on the key components of our system to gain detailed insights into the relationship between component-wise behavior and various parameters.

\section{Related Work}

NLG for Dialogue Response Generation There has been a body of work on neural NLG models, adopting various architectures, such as RNNs (Wen et al., 2015), attention RNNs (Dušek and Jurčíček, 2016), SC-LSTM (Wen et al., 2016), T2G2 (Kale and Rastogi, 2020), AdapterCL (Madotto et al., 2020) and associated variants (Tran and Le Nguyen, 2017; Tran et al., 2017). Despite the improved flexibility and naturalness over template-based methods, neural approaches require large amounts of annotated data to reach good performance.

Data Augmentation Data augmentation has been widely applied to a variety of NLP tasks, including sentence classification (Xie et al., 2020), natural language inference (Hu et al., 2019) and spoken language understanding (Li et al., 2019; Quan and Xiong, 2019; Zhao et al., 2019). Prior approaches for text data utilized back-translation (Sennrich et al., 2016; Edunov et al., 2018), c-BERT word replacement (Jiao et al., 2020), mixed labels and representations (Guo et al., 2019; Chen et al., 2020) and paraphrase data (Gao et al., 2020). However, the range of augmented data will be inherently limited, particularly in few-shot learning settings due to the nature of prior approaches, which only leverages in-domain data. In contrast, we take a rarely explored approach, tapping into a wealth of opendomain text that covers almost all topics. Recently, Du et al. (2021) proposed a self-training method

\footnotetext{
${ }^{1}$ Since SGD accounts for a large portion of the existing MR-to-Text data that SC-GPT utilized in training, we could not apply AUGNLG to SC-GPT for the FEWSHOTSGD task.
}

to augment data for NLU tasks by retrieving sentences from data crawled on the web. However, their method cannot be directly applied to the NLG problem since it does not yield MR annotations. Our approach, in contrast, generates MR-to-Text data by jointly employing a self-trained neural retrieval model with a few-shot learned NLU model.

\section{Few-shot Transfer Learning for NLG}

The goal of NLG is to translate an MR $\mathcal{A}$ into its natural language response $x=\left[x^{1}, \ldots, x^{T}\right]$, where $x^{i}$ is the $i$ th token in the sequence $x$ and $T$ is the sequence length. $\mathcal{A}$ is defined as the combination of intent $\mathcal{I}$ and slot-value pairs $\left\{\left(s_{i}, v_{i}\right)\right\}_{i=1}^{P}$ :

$$
\mathcal{A}=\left\{\mathcal{I},\left(s_{1}, v_{1}\right), \ldots,\left(s_{P}, v_{P}\right)\right\},
$$

where the intent stands for the illocutionary type of the system action while slot-value pairs indicate category names and their values to embed in the utterance. For example, in the MR, inform (food = chinese ; price $=$ cheap), inform is the intent, food and price are two slot keys and chinese and cheap are the corresponding slot values.

Given in-domain MR-to-Text data $\mathbb{D}=$ $\left\{\left(\mathcal{A}_{n}, x_{n}\right)\right\}_{n=1}^{N}$ for training, where $N$ is the number of examples, a statistical neural language model parameterized by $\theta$ is adopted to characterize the conditional probability $p_{\theta}(x \mid \mathcal{A})$. By adopting the chain rule on auto-regressive generation, the joint probability of $x$ conditioned on $\mathcal{A}$ is decomposed as $\prod_{t=1}^{T} p_{\theta}\left(x^{t} \mid x^{<t}, \mathcal{A}\right)$. The training process, i.e. the learning of $\theta$, is then defined as maximizing the log-likelihood of the conditional probabilities over the entire training dataset:

$$
\mathcal{L}_{\theta}(\mathbb{D})=\sum_{n=1}^{|\mathbb{D}|} \log p_{\theta}\left(x_{n} \mid \mathcal{A}_{n}\right) .
$$

In the few-shot learning setup, the number of training examples $N$ is extremely small (e.g. $\leq 50$ ), which easily leads to non-fluent generated sentences with many grammar mistakes or missing pieces of information. In order to combat the data sparseness problem, inspired by prior transfer learning approaches, we introduce a three-step pipeline to gradually evolve a general large-scale language model to a domain-specific NLG model (shown in Figure 2): (1) pre-training a base language model with massive amounts of text, (2) NLG-specific continued pre-training with auto-augmented MRto-Text data, and (3) final fine-tuning with the limited in-domain MR-to-Text ground-truth data. 


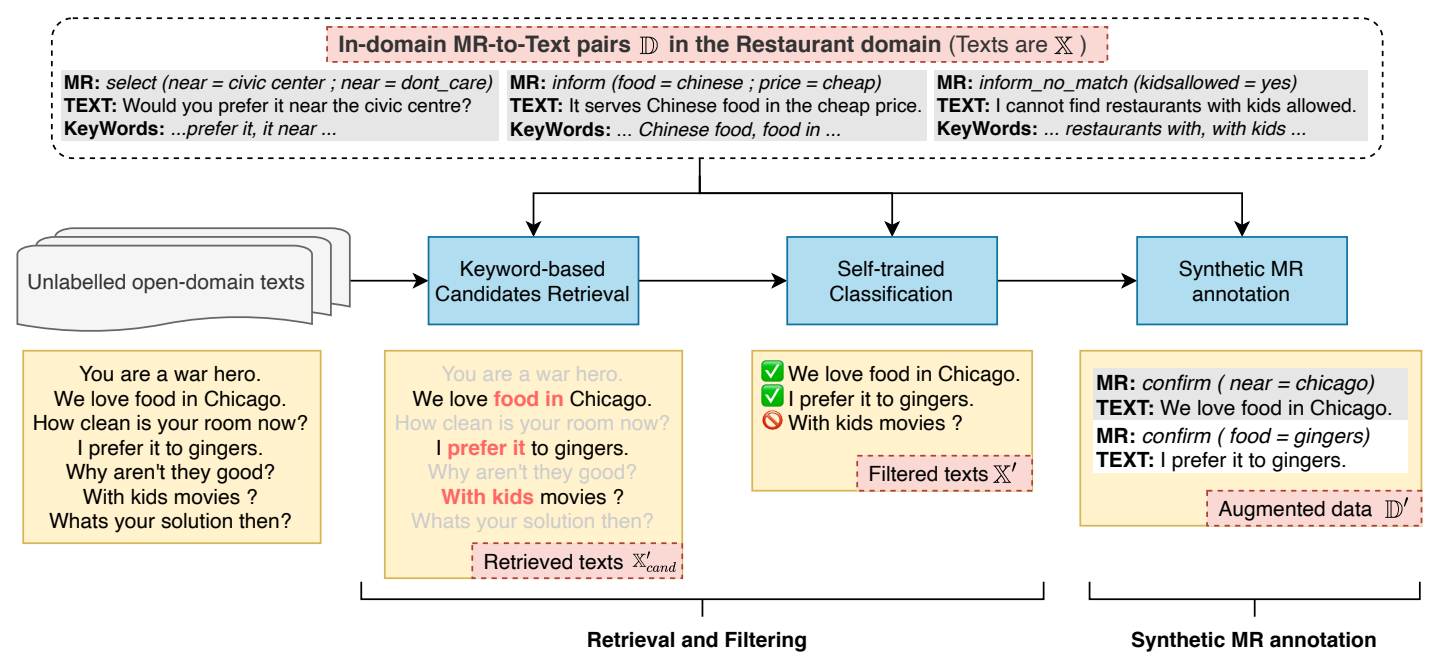

Figure 3: The overall pipeline for MR-to-Text data augmentation.

Specifically, in Step (1), we adopt GPT-2 (Radford et al., 2019) as our base language model since GPT-2 has demonstrated a remarkable performance on auto-regressive text generation tasks, which is close to MR-to-Text generation, in a variety of domains. However, GPT-2 is pre-trained on OpenWebText and the language style and topics thereof are quite different from those of daily conversations in a target domain. Furthermore, the generation task in NLG is conditioned on the input $\mathrm{MR}$, as opposed to the unconditioned generation of the underlying GPT-2 pre-training task. Thus, to bring the model a step closer to the final NLG model in the target domain, in Step (2), we continuously pre-train the GPT-2 model on an automatically constructed set of augmented MR-to-Text pairs $\mathbb{D}^{\prime}=\left\{\left(\mathcal{A}_{m}, x_{m}\right)\right\}_{m=1}^{M}$, where $M$ is the number of augmented examples, which is much larger than the amount of in-domain ground-truth data. Data augmentation is achieved by retrieving a large amount of relevant text from Reddit (Henderson et al., 2019) with a self-trained neural retrieval model and then synthesizing MRs with a few-shot learned NLU model. The details of data augmentation is described in Section 4. Finally, in Step (3), we fine-tune the NLG model on a limited amount of in-domain ground-truth MR-to-Text pairs $\mathbb{D}$ for a final adaptation.

\section{Data Augmentation}

The data augmentation procedure aims to construct a large amount of MR-to-Text pairs $\mathbb{D}^{\prime}$ from open-domain texts that are relevant to the in-domain ground-truth MR-to-Text pairs $\mathbb{D}$. The augmentation process consists of two stages: (1) retrieving keyword-matching utterances and filtering out domain-irrelevant instances, (2) generating synthetic MR annotations. Figure 3 illustrates the overall pipeline with some examples. For further analysis and studies, we release the data from all intermediate steps for each domain at https://github.com/Xinnuoxu/ AugNLG/tree/master/augmented_data.

\subsection{Retrieval and Filtering}

The utterance retrieval and filtering procedure consists of three steps: (1) keyword extraction that collects n-gram keywords from all in-domain utterances $\mathbb{X}=\left\{x_{n}\right\}_{n=1}^{N}$; (2) keyword-based retrieval that searches the open-domain texts for utterances that match any keywords extracted in the previous step, yielding a set of utterances $\mathbb{X}_{\text {cand }}^{\prime}$; (3) self-trained neural classifier that filters out some retrieved utterances that are semantically irrelevant to the target domain. After the filtering, we form an augmented set of utterances $\mathbb{X}^{\prime}$ with the unfiltered utterances.

Keywords Extraction. To efficiently extract keywords, we first gather all n-gram phrases that appear in $\mathbb{X}$. Since some phrases are too general to be effective, e.g. "I cannot", "is your", we use TFIDF scores to measure the specificity of a phrase (see Appendix A for more detail). We first rank the collected n-grams according to their TF-IDF scores and filter out those n-gram phrases with relatively low TF-IDF score.

Keyword-based Retrieval. Having extracted the keywords, we retrieve utterances from the opendomain utterance pool that contains at least one 


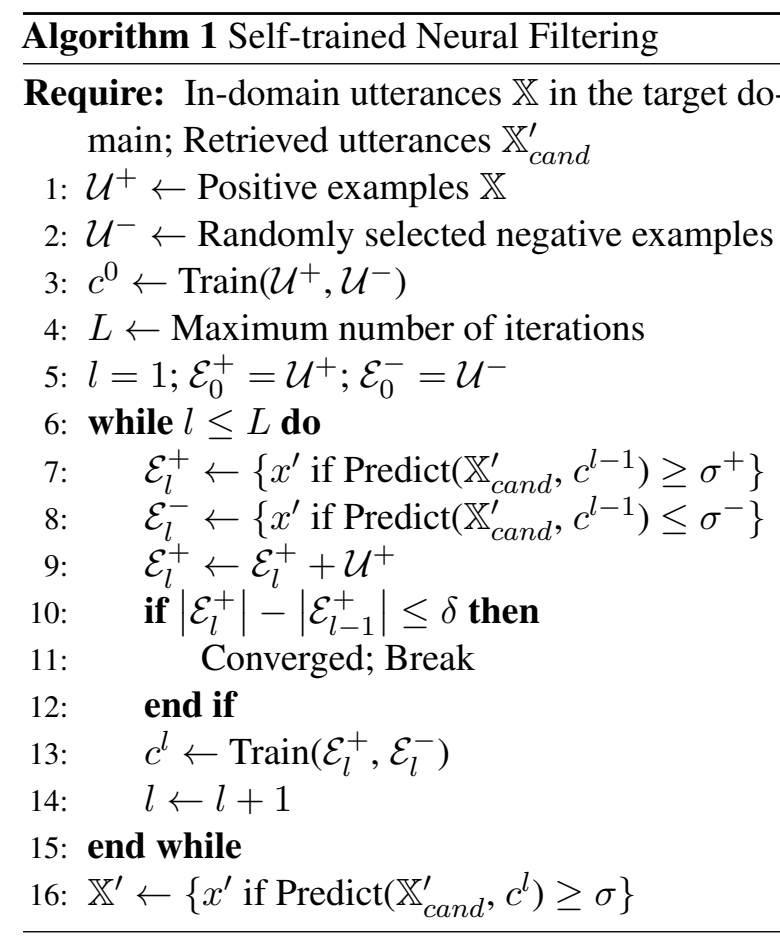

extracted keyword in it. The aim of this step is to source a large amount of domain-relevant utterances $\mathbb{X}_{\text {cand }}^{\prime}$ based on the surface-level overlap.

Self-trained Neural Filtering. Although the keyword-based retrieval is efficient, the retrieved utterances $\mathbb{X}_{\text {cand }}^{\prime}$ can be quite noisy since an n-gram keyword only matches some part of the utterance, failing to detect the existence of irrelevant pieces in other parts. For example, in Figure 3, even though the utterance "With kids movies?" contains the keyword "with kids", it is irrelevant to the target domain Restaurant given the word movies. Thus, we introduce a self-trained neural classifier to filter out domain-irrelevant utterances from $\mathbb{X}_{\text {cand }}^{\prime}$ by considering the semantic representation of an entire utterance and yield a domain-relevant set $\mathbb{X}^{\prime}$.

The algorithm of the self-training and filtering process is listed in Algorithm 1. We adopt a BERT (Devlin et al., 2019) model with a binary classification layer atop as the base model and then train the classifier with in-domain utterances $\mathbb{X}$ and randomly selected open-domain utterances ${ }^{2}$, serving as positive and negative examples $\left(\mathcal{U}^{+}\right.$and $\left.\mathcal{U}^{-}\right)$, respectively. After that, the self-training and filtering cycle starts. At each iteration, we make predictions on the utterances in $\mathbb{X}_{\text {cand }}^{\prime}$ with the classifier

\footnotetext{
${ }^{2}$ All utterances in $\mathbb{X}_{\text {cand }}^{\prime}$ are excluded from the opendomain utterance pool. To balance the precision and recall, we control the size of the initial negative set such that $\left|\mathcal{U}^{-}\right|=\lambda_{1} \cdot\left|\mathcal{U}^{+}\right|$, where $\lambda_{1}=10$.
}

trained in the previous iteration. All utterances with a score over the threshold $\sigma^{+}$, together with the in-domain utterances $\mathbb{X}$, are then taken as a new set of positive examples $\mathcal{E}^{+}$, whereas all utterances with a score less than the threshold $\sigma^{-}$are collected as a new set of negative examples $\mathcal{E}^{-}$. The self-training loop terminates if either the increment of positive examples at the last iteration is less than the threshold $\delta$ or the iterations is over the pre-defined maximum number of iterations. Otherwise, a new classifier is trained on $\mathcal{E}^{+}$and $\mathcal{E}^{-}$and the algorithm keeps going on the loop. Once the loop terminated, we label all utterances in $\mathbb{X}_{\text {cand }}^{\prime}$ with the classifier from the last iteration. Finally, we build a domain-relevant set of augmented utterances $\mathbb{X}^{\prime}$ by taking all utterances with a score over the threshold $\sigma{ }^{4}$

\subsection{Synthetic MR Annotation}

Having built the domain-relevant set of augmented utterances $\mathbb{X}^{\prime}$, we now proceed to synthesize MR labels to produce a complete MR-to-Text dataset $\mathbb{D}^{\prime}$. To this end, we build a few-shot NLU model by fine-tuning a BERT model with in-domain groundtruth data. To put the data in the right format for the NLU task, we take MRs and utterances as labels and model inputs, respectively. Each token is annotated with the slot name if it is a part of the associated slot value and the final hidden state of the special token [CLS] is used to predict the intent (see Figure 5 in Appendix B). Finally, we generate an MR-to-Text dataset $\mathbb{D}^{\prime}$ by concatenating the utterances in $\mathbb{X}^{\prime}$ with the synthetic MR labels predicted by the few-shot NLU model.

\section{Experimental Setup}

\subsection{Dataset}

Fewshot NLG Data FewShotWOZ is a fewshot NLG benchmark, built upon RNNLG and MultiWOZ (Budzianowski et al., 2018). In each domain, MR-to-Text pairs are grouped according to their delexicalized MRs (i.e. slot values being masked) and a training set is created by taking a pair each from 50 random groups and then the rest are taken as the test set. We also construct a new dataset FEWSHOTSGD by applying the same

\footnotetext{
${ }^{3}$ To guarantee the precision of the positive examples, we use $\sigma^{+}=0.99$ and $\sigma^{-}=0.5$. Also, we sub-sample negative examples such that $\left|\mathcal{E}^{-}\right|=\lambda_{2} \cdot\left|\mathcal{E}^{+}\right|$, where $\lambda_{2}=5$.

${ }^{4}$ To harvest a large amount of utterances, we set the threshold $\sigma$ to 0.5 .
} 


\begin{tabular}{l|cc}
\hline Statistics & -WOZ & -SGD \\
\hline \# Domains & 7 & 16 \\
Avg. \# Intents & 8.14 & 6.44 \\
Avg. \# Slots & 16.2 & 11.3 \\
Avg. \# Delex MRs in Training & 50 & 33 \\
Avg. \# Delex MRs in Testing & 473 & 31 \\
Avg. \# Training Instances & 50 & 35 \\
Avg. \# Test Instances & 473 & 5618 \\
Avg. \# Test Instances per MR & 1.14 & 472.9 \\
Avg. \# Test Novelty uni-gram (\%) & 12.97 & 23.90 \\
Avg. \# Test Novelty bi-gram(\%) & 44.42 & 65.29 \\
Avg. \# Test Novelty tri-gram(\%) & 68.20 & 84.44 \\
Avg. \# Test Novelty four-gram(\%) & 82.70 & 92.75 \\
\hline Avg. \# Keywords (K) & 0.20 & 0.12 \\
Avg. \# Retrieved Utterances (K) & 854.8 & 731.3 \\
Avg. \# Augmented Pairs (K) & 34.0 & 25.6 \\
Avg. \# Delex. MRs in Aug. Pairs (K) & 2.12 & 0.57 \\
\hline
\end{tabular}

Table 1: Comparison of FEwShotWOZ and FEWSHOTSGD. The bottom section shows the statistics for augmented data. The unit for all statistics in the bottom section is thousand $(\mathrm{K})$.

preparation steps to the SGD corpus. The comparison of FEWSHOTWOZ and FEWSHотSGD is presented in the top section in Table 1. Comparing to FEwSHOтWOZ, FEwSHOtSGD has (1) more domains, (2) less intents, slots and delexicalized $\mathrm{MRs}^{5}$ (3) more testing examples for each delexicalized MR, (4) more novel n-grams ${ }^{6}$ in test utterances.

Augmented Data Since Reddit has shown to provide natural conversational English data, we adopt Reddit (Henderson et al., 2019) as the open-domain utterance pool after filtering for utterances of length between 2 and 40, totalling about 0.7B utterances. The average number of extracted keywords, retrieved utterances, final augmented MR-to-Text pairs and delexicalized MRs over all domains in FEWSHOTWOZ and FEWSHOTSGD are shown in the bottom section of Table 1 . The detailed breakdowns of each domain are listed in Table 9 and Table 10 in Appendix C.

\subsection{Evaluation Metrics}

Following Wen et al. (2015) and Peng et al. (2020), we use BLEU score and Slot Error Rate (ERR) for automatic evaluation. BLEU score measures the surface-level similarity between generated responses and human-authored references. Whereas,

\footnotetext{
${ }^{5}$ Note that, the average number of delexicalized MRs in the training set is 33 , which means the number of training examples in some domains are less than 50 .

${ }^{6}$ The novelty is calculated by dividing the number of $n$ grams in the test set that does not appear in the training set by the number of $n$-grams in the test set.

${ }^{7}$ https://github.com/pengbaolin/SC-GPT.
}

ERR measures the semantic alignment in terms of slot-value insertion and omission. Specifically, ERR $=(p+q) / M$, where $M$ is the total number of slots in the MR and $p, q$ are the number of missing and redundant slots in the surface realisation. Since the SGD dataset does not provide enough information to compute ERR, we report ERR only on FEwSHOTWOZ.

\subsection{Systems}

We apply our data augmentation approach AUGNLG to two baseline systems,

- FT-GPT GPT-2 is directly fine-tuned on the in-domain ground-truth MR-to-Text data. We introduce AUGNLG-FT, which further pretrains GPT-2 on the augmented MR-to-Text data and performs a final fine-tuning on the in-domain data.

- SC-GPT (Peng et al., 2020) further pre-trains GPT-2 on existing MR-to-Text data borrowed from other NLG corpora and fine-tunes on the in-domain data. We introduce AUGNLGSC, which pre-trains GPT-2 on both existing MR-to-Text data and automatically augmented data, and finally fine-tunes on the indomain data.

\section{Results}

FEWSHOTWOZ Table 2 reports the results on FEWSнотWOZ. AugNLG-FT substantially outperforms FT-GPT across all domains in both BLEU and ERR. Similarly, AUGNLG-SC performs better than SC-GPT and achieves the state-of-theart performance in most domains. Remarkably, AUGNLG-FT achieves a competitive performance with SC-GPT in many domains without leveraging any existing MR-to-Text data. It even outperforms SC-GPT in "TV" and "Attraction" domain in both BLEU and ERR.

FEWSHOTSGD Table 3 shows the results in FEwSHOTSGD. Due to the higher novelty of the test examples and the smaller amount of training examples (see Avg. \# Test Novelty n-gram and \# Training Instances in Table 1), FT-GPT performs worse than on FEWSHOTWOZ. This indicates that the few-shot settings on FEWSHOTSGD are even more challenging. But AUGNLG-FT managed to outperform FT-GPT by a large margin via the continued pre-training on the augmented examples. 


\begin{tabular}{|c|c|c|c|c|c|c|c|}
\hline Model & $\begin{array}{l}\text { Restaurant } \\
\text { BLEU ERR }\end{array}$ & $\begin{array}{c}\text { Laptop } \\
\text { BLEU ERR }\end{array}$ & \begin{tabular}{c|} 
Hotel \\
BLEU ERR
\end{tabular} & $\begin{array}{c}\text { TV } \\
\text { BLEU ERR }\end{array}$ & $\begin{array}{l}\text { Attraction } \\
\text { BLEU ERR }\end{array}$ & \begin{tabular}{c|} 
Train \\
BLEU ERR
\end{tabular} & $\begin{array}{c}\text { Taxi } \\
\text { BLEU ERR }\end{array}$ \\
\hline FT-GPT & $28.15 \quad 15.87$ & $\begin{array}{ll}28.83 & 11.82\end{array}$ & $\begin{array}{|ll|}36.51 & 14.29\end{array}$ & $\begin{array}{ll}33.73 & 9.28\end{array}$ & $\begin{array}{|ll|}17.45 & 22.83 \\
\end{array}$ & $\begin{array}{ll}13.06 & 25.59\end{array}$ & 14.84 \\
\hline AUGNLG-FT & $32.16 \quad 4.79$ & $33.64 \quad 5.14$ & $36.99 \quad 9.89$ & $34.80 \quad 6.92$ & $20.61 \quad 13.58$ & $14.95 \quad 10.64$ & $16.70 \quad 10.71$ \\
\hline SC-GPT & $30.48 \quad 6.89$ & $\begin{array}{lll}33.51 & 5.38\end{array}$ & $\mathbf{3 8 . 3 0} 8.24$ & 33.827 .32 & $22.24 \quad 16.62$ & 17.068 .82 & 19.214 .76 \\
\hline AUGNLG-SC & $\begin{array}{ll}34.20 & 2.99\end{array}$ & $\begin{array}{ll}34.32 & 2.83 \\
\end{array}$ & $34.96 \quad 6.59$ & $34.99 \quad 5.53$ & 22.5010 .40 & $16.35 \quad \mathbf{6 . 1 3}$ & $17.81 \quad \mathbf{3 . 5 7}$ \\
\hline
\end{tabular}

Table 2: Evaluation results on FEWSHOTWOZ (BLEU $\uparrow$, ERR $\downarrow$ ). Note that, the SC-GPT model reported here was pre-trained and fine-tuned using the code and only the SGD data shared by the original authors ${ }^{7}$.

\begin{tabular}{l|cccccccc}
\hline Model & Restaurants & Hotels & Flights & Calendar & Banks & Weather & Buses & Events \\
\hdashline FT-GPT & 08.98 & 08.84 & 12.18 & 05.27 & 06.09 & 10.52 & 07.77 & 09.17 \\
AUGNLG-FT & $\mathbf{1 7 . 8 3}$ & $\mathbf{1 7 . 2 3}$ & $\mathbf{1 7 . 5 8}$ & $\mathbf{1 0 . 4 5}$ & $\mathbf{0 8 . 9 4}$ & $\mathbf{1 3 . 7 5}$ & $\mathbf{1 4 . 2 6}$ & $\mathbf{1 8 . 6 8}$ \\
\hline \hline Model & Homes & Media & Movies & Music & Rentalcars & Ridesharing & Services & Travel \\
\hdashline FT-GPT & 03.75 & 03.17 & 10.05 & 05.79 & 06.79 & 13.87 & 09.79 & 02.08 \\
AUGNLG-FT & $\mathbf{1 2 . 2 7}$ & $\mathbf{0 8 . 6 2}$ & $\mathbf{1 1 . 9 6}$ & $\mathbf{1 2 . 7 6}$ & $\mathbf{1 3 . 3 2}$ & $\mathbf{1 5 . 5 4}$ & $\mathbf{1 6 . 8 2}$ & $\mathbf{1 4 . 3 5}$ \\
\hline
\end{tabular}

Table 3: Evaluation results in BLEU on FEwSHOTSGD.

Qualitative Evaluation Table 4 compares some generated utterances by different models on FEWSHOTWOZ (examples in FEWSHOTSGD are shown in Table 16 in Appendix E). Both FT-GPT and SC-GPT are prone to omit important slots. Comparing to SC-GPT, FT-GPT tends to overgenerate and introduces hallucinations. However, AUGNLG and AUGNLG-SC managed to generate fluent, natural text while precisely reflecting the the input MR. We further examined 70 randomly sampled utterances generated by AUGNLG-SC, whose BLEU scores are lower than those generated by SCGPT, in the "Hotel", "Train" and "Taxi" domain to understand some potential factors causing the lower BLEU scores We found that the lower BLEU scores are mainly driven by BLEU penalizing semantically correct paraphrases due to the nature of BLEU only checking surface-level matches. Some examples of such penalization are provided in $\mathrm{Ta}-$ ble 15 in Appendix E. Only 7 out of the 70 manually checked examples generated by AUGNLG-SC are actually worse than SC-GPT. ${ }^{8}$

In sum, the results (1) verify the effectiveness of complementing existing transfer learning methods with our novel data augmentation approach; (2) reveal that automatically augmented MR-to-Text data alone can lead to a competitive performance, previously only achieved with existing MR-to-Text data. Since existing MR-to-Text data is not a scalable data source, our approach brings more practical values to real-world applications; (3) indicate that

\footnotetext{
${ }^{8}$ We also examined 70 randomly sampled utterances generated by AUGNLG-SC, whose BLEU scores are equal/higher than those generated by SC-GPT. Among these examples, 35 examples are actually better and 7 examples are worse than the SC-GPT generations.
}

leveraging augmented MR-to-Text data on top of existing MR-to-Text data yields a new SOTA performance on the benchmark test.

\section{$7 \quad$ In-depth Analysis}

In this section, we provide comprehensive analysis results on the key components and parameters of our system to gain detailed insights: (1) intrinsic evaluation on augmented data, (2) influence of NLU quality, and (3) performance trends over varying amounts of augmented data.

\subsection{Intrinsic Evaluation on Augmented Data}

For intrinsic evaluation of augmented data, we first introduce four metrics:

- MR coverage (MR Cov.) evaluates the coverage of delexicalized MRs of the test set in the augmented set:

$$
\text { MR Cov. }=\frac{\# \text { delexicalized MRs } \in \mathbb{A}^{\prime} \cap \mathbb{A}_{\text {test }}}{\# \text { delexicalized MRs } \in \mathbb{A}_{\text {test }}},
$$

where $\mathbb{A}^{\prime}$ and $\mathbb{A}_{\text {test }}$ denote delexicalized MRs in the augmented set and the test set, respectively. Higher MR Cov. values indicate that more delexicalized MRs of the test set appear in the augmented set.

- Slot coverage (SL Cov.) evaluates the coverage of slot keys of the test set in the augmented set.

- Language model perplexity (PPL) is the perplexity of augmented utterances calculated by a GPT-2 language model fine-tuned on the test set. Lower $P P L$ values indicate that the distribution of augmented utterances is close to that of the test utterances.

- Average n-gram novelty (Nvt.) N-gram novelty measures the fraction of the $\mathrm{n}$-grams in the test set 


\begin{tabular}{|c|c|}
\hline \multicolumn{2}{|r|}{ Domain: Restaurant } \\
\hline Input MR & inform(name=marlowe; goodformeal=dinner; area=mission bay) \\
\hline Reference & marlowe serves dinner in the mission bay area. \\
\hline FT-GPT & there is a restaurant marlowe in the mission bay area called dinner, it is good for dinner, a good area. \\
\hline SC-GPT & marlowe is a good restaurant in mission bay that serves dinner. \\
\hline AUGNLG-FT & marlowe is a good restaurant that serves good dinner in mission bay. \\
\hline AUGNLG-SC & marlowe is good for dinner in mission bay. \\
\hline \multicolumn{2}{|r|}{ Domain: Laptop } \\
\hline Input MR & inform (name $=$ tecra proteus $23 ;$ type $=$ laptop $;$ battery $=9$ hour $;$ memory $=4$ gb;isforbusiness $=$ false $)$ \\
\hline Reference & the tecra proteus 23 laptop has a 9 hour battery life with 4 gb memory but is not for business computing. \\
\hline FT-GPT & tecra proteus 23 laptop with 9 hour battery and $4 \mathrm{gb}$ memory. $\%$ miss $\{$ isforbusiness $=$ false $\}$ \\
\hline SC-GPT & tecra proteus 23 laptop with 4 gb memory and 9 hour battery. $\%$ miss $\{$ isforbusiness $=$ false $\}$ \\
\hline AUGNLG-FT & the tecra proteus 23 laptop has a 9 hour battery, $4 \mathrm{gb}$ of memory, and is not for business computing. \\
\hline AUGNLG-SC & tecra proteus 23 laptop - 9 hour battery - 4 gb memory - not used for business computing. \\
\hline \multicolumn{2}{|r|}{ Domain: $T V$} \\
\hline Input MR & inform (name $=$ pontus $45 ;$ type $=t v ;$ ecorating $=a+;$ screensizerange $=$ medium; hdmiport $=2)$ \\
\hline Reference & the pontus 45 tv has a a+ eco rating and a medium screen size and 2 hdmi ports. \\
\hline FT-GPT & the pontus 45 tv has 2 hdmi ports, a medium screen size, and 2 screensize. $\%$ miss $\{$ ecorating $=a+\}$ \\
\hline SC-GPT & the pontus 45 tv has a medium screen size and 2 hdmi ports. $\%$ miss $\{$ ecorating $=a+\}$ \\
\hline AUGNLG-FT & the pontus 45 tv has a+ eco rating, 2 hdmi ports, and a medium screen size. \\
\hline AUGNLG-SC & the pontus $45 \mathrm{tv}$ has a medium screen size of 2 hdmi ports and a+ eco rating. \\
\hline \multicolumn{2}{|r|}{ Domain: Attraction } \\
\hline Input MR & inform (area $=$ north;price $=$ no entrance fee $;$ type $=$ park; phone $=01223$ ) \\
\hline Reference & yes, it's a park located in the north area of town and has no entrance fee. the phone number is 01223 . \\
\hline FT-GPT & yes, there is a free entrance fee of $£ 1$. phone 01223 for more information. $\%$ miss $\{$ area $=$ north $\}$ \\
\hline SC-GPT & no problem. 01223 is the phone number and the park is north. the entrance fee is free. \\
\hline AUGNLG-FT & yes, the entrance fee is no entrance fee. the park is in the north. phone is 01223 . \\
\hline AUGNLG-SC & yes, the park is in the north. no entrance fee. phone number 01223 . \\
\hline
\end{tabular}

Table 4: Example utterances generated by different models on FEWSHOTWOZ (Better viewed in color). Errors are shown in three colors. The red text starting with "\%" denotes omission. The blue text indicates hallucination. The green text means non-fluent generation.

\begin{tabular}{l|ccccccc}
\hline Metrics & Re & La & Ho & TV & At & Tr & Ta \\
\hdashline MR Cov. $\uparrow$ & .70 & .21 & .71 & .40 & .66 & .44 & .59 \\
SL Cov. $\uparrow$ & 1.0 & .95 & 1.0 & .94 & .89 & .92 & .86 \\
\hline
\end{tabular}

Table 5: Augmented data evaluation of MR Cov. and SL Cov. on FEWSHOTWOZ. The domain names are represented by the first two letters.

\begin{tabular}{l|llllllll}
\hline Metrics & Re & Ho & Fl & Ca & Ba & We & Bu & Ev \\
\hdashline MR Cov $\uparrow$ & .80 & .72 & .66 & .65 & .43 & .70 & .58 & .80 \\
SL Cov $\uparrow$ & .92 & .85 & .89 & .75 & .57 & .86 & .88 & .93 \\
\hline \hline Metrics & Ho & Me & Mo & Mu & Re & Ri & Se & Tr \\
\hdashline MR Cov $\uparrow$ & .59 & .58 & .74 & .67 & .81 & .77 & .88 & .55 \\
SL Cov $\uparrow$ & .75 & .67 & .80 & .75 & .80 & .78 & .93 & .71 \\
\hline
\end{tabular}

Table 6: Augmented data evaluation of MR Cov. and SL Cov. on FEWSHotSGD. The domain names are represented by the first two letters.

that do not appear in the augmented set:

$$
\mathrm{N} \text {-gram novelty }=1-\frac{\# \text { n-grams } \in \mathbb{X}^{\prime} \cap \mathbb{X}_{\text {test }}}{\# \text { n-grams } \in \mathbb{X}_{\text {test }}}
$$

where $\mathbb{X}^{\prime}$ and $\mathbb{X}_{\text {test }}$ denote utterances in the augmented set and test set, respectively. Lower $N v t$. values indicate that more n-grams of the test set appear in the augmented set. We consider from 1-grams to 4-grams and report the average value.

The results of MR Cov. / SL Cov. on FEWSHOT-
WOZ and FEwSHOTSGD are shown in Table 5 and Table 6, respectively. SL Cov. achieves $70 \%$ in most domains on both datasets while MR Cov. has a wide range of values across domains. Noteworthily, Table 6 strongly correlates with Table 3 - "Banks" and "Media" domains are worse than other domains in both coverage metrics and NLG performance. On the other hand, "Restaurants" and "Events" domains are better than the others in both aspects. Although we do not see the same pattern on FEwSнотWOZ, it could be attributed to the large variance in the number of delexicalized MRs in each domain (see Table 2 in (Peng et al., 2020)).

The results of PPL and Nvt. on FEWSHOTWOZ are shown in Table 7 . We compare the augmented data $(A U G)$ with the existing MR-to-Text data $(E X$ $I S T)$. The top section shows that $A U G$ achieves lower $P P L$ values in all seven domains compared to EXIST. The bottom section again demonstrates that $A U G$ achieves lower $N v t$. values in most domains. However, in the "Train" and "Taxi" domains EXIST attains lower novelty values, which matches the results in Table 2, SC-GPT outperforming AUGNLG-SC in these two domains. ${ }^{9}$

\footnotetext{
${ }^{9}$ Detailed breakdowns of novelty scores from 1-grams to 4-grams are provided in Table 11 in Appendix C. The Nvt. re-
} 


\begin{tabular}{c|l|ccccccc}
\hline Metrics & Data & Restaurant & Laptop & Hotel & TV & Attraction & Train & Taxi \\
\hline PPL $\downarrow$ & EXIST & 04.14 & 22.92 & 04.09 & 19.53 & 08.28 & 09.04 & 06.74 \\
& AUG & $\mathbf{0 3 . 4 8}$ & $\mathbf{0 8 . 4 6}$ & $\mathbf{0 2 . 8 9}$ & $\mathbf{0 5 . 7 7}$ & $\mathbf{0 4 . 7 3}$ & $\mathbf{0 6 . 7 7}$ & $\mathbf{0 6 . 7 2}$ \\
\multirow{2}{*}{ Nvt. $(\boldsymbol{\%}) \downarrow$} & EXIST & 57.36 & 71.11 & 55.21 & 72.34 & 55.37 & $\mathbf{5 3 . 4 5}$ & $\mathbf{4 6 . 9 4}$ \\
& AUG & $\mathbf{5 4 . 5 0}$ & $\mathbf{5 0 . 7 3}$ & $\mathbf{4 8 . 3 9}$ & $\mathbf{4 4 . 9 3}$ & $\mathbf{3 9 . 8 3}$ & 56.24 & 55.38 \\
\hline
\end{tabular}

Table 7: Language Model perplexity (PPL) and average n-gram novelty (Nvt.) on augmented data.
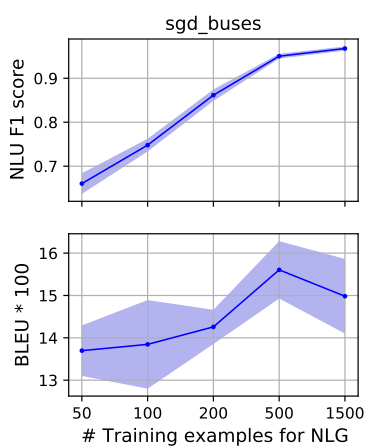
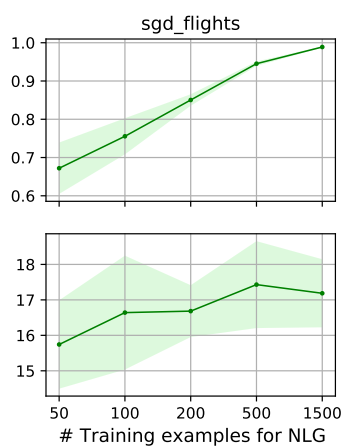
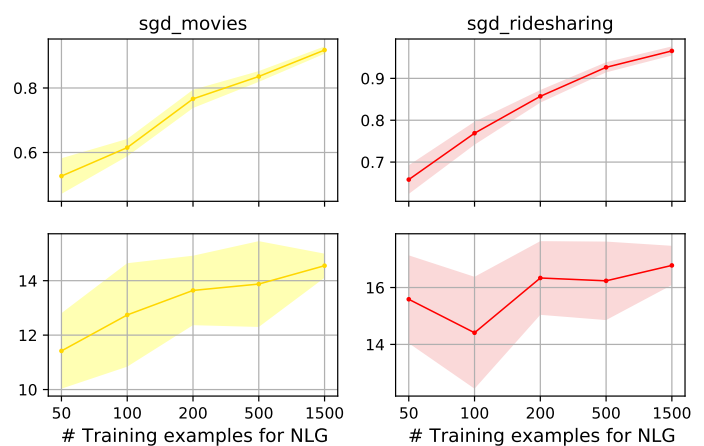

Figure 4: The influence of NLU on four domains in FEwSHOTSGD. The top row shows NLU F1 scores with 50,100,200, 500, 1500 training examples. The bottom row shows the BLEU scores of AUGNLG-FT pre-trained using these NLU models. All experiments are repeated for 5 times with different samples.

\subsection{Influence of NLU}

Few-shot NLU performance Since few-shot NLU models are a key component of our system, we report their performance in F1 score. For each domain, we evaluate the few-shot NLU model on the Text-to-MR test set, prepared in Section 4.2. The average F1 over all domains on FEwSHOTWOZ and FEWSHOTSGD are 0.77 and 0.68 , respectively. A further breakdown over the domains are provided in Table 13 and Table 14 in Appendix D.

Influence of NLU Quality The mediocre NLU performance on FEWSHOTSGD leads to the following research question: can better NLU models boost NLG performance? To answer this question, we select four domains from FEWSHOTSGD with relatively low NLU performance: "Buses (0.63)", "Flights (0.74)", "Movies (0.44)", and Ridesharing (0.63). In each domain, we construct a new test set by randomly sampling 500 MR-to-Text pairs from the original test set, and take the rest as the NLU training pool. To obtain NLU models of varying quality, we train a set of models while varying the amount of training data with stratified sampling. The top row in Figure 4 clearly shows that F1 score increases in proportion to the training size, reaching 0.95 in $\mathrm{F} 1$ in all four domains. We then annotate the augmented utterances with different

sults on FEWSHOTSGD are shown in Table 12 in Appendix C, demonstrating similar trends.

\begin{tabular}{c|c|ccccc}
\hline Do & Mod & $\mathbf{5 0}$ & $\mathbf{1 0 0}$ & $\mathbf{2 0 0}$ & $\mathbf{5 0 0}$ & $\mathbf{1 5 0 0}$ \\
\hline \hline \multirow{2}{*}{$\mathrm{Bu}$} & FT & 7.87 & 10.38 & 15.21 & 21.83 & 24.91 \\
& AUG & 14.37 & 15.36 & 17.06 & 22.18 & 24.98 \\
$\mathrm{Fl}$ & FT & 10.40 & 12.93 & 19.91 & 25.97 & 29.18 \\
& AUG & 14.07 & 15.50 & 21.55 & 25.38 & 26.62 \\
$\mathrm{~N}$ & FT & 13.30 & 16.13 & 21.99 & 29.76 & 34.04 \\
& AUG & 17.13 & 17.55 & 23.68 & 29.14 & 33.55 \\
$\mathrm{Ni}$ & FT & 12.32 & 16.99 & 23.25 & 27.99 & 29.02 \\
& AUG & 17.18 & 22.06 & 24.76 & 26.87 & 28.60 \\
\hline
\end{tabular}

Table 8: BLEU scores for FT-GPT (FT) and AUGNLGFT (AUG) with different training sizes (50, 100, 200, 500, 1500). "Bu", "Fl", "Mo" and "Ri" are short for the domain names "Buses", "Flights", "Movies", "Ridesharing". All experiments are repeated for 5 times with different samples.

NLU models and pre-train the NLG models with the augmented MR-to-Text data updated with new MR labels. Finally, we fine-tune the NLG models on the in-domain training set $\mathbb{D}$ and perform evaluation on the newly constructed 500 test set. The bottom row in Figure 4 confirms that there is a general proportional relationship between the performances of NLU and NLG.

\subsection{Varying Amounts of Augmentation}

Lastly, we investigate the relationship between the amount of in-domain ground-truth data and the effect of augmentation. As in the previous section, we build new test sets by randomly taking 500 examples and vary the size of training set to train both NLU and NLG models. Table 8 shows that, in all four domains, the performance difference 
between AUGNLG-FT and FT-GPT culminates at the smallest training set and gradually diminishes as more training data become available.

\section{Conclusion}

In this paper, we proposed AUGNLG, a novel data augmentation approach that combines a self-trained retrieval model with a few-shot learned NLU, to automatically create MR-to-Text data from opendomain texts. Experimental results verify the effectiveness of our approach by establishing new SOTA performances on two benchmark tests. More importantly, we showed how our approach complements the previous SOTA approach, which hinges on unscalable data sources, with unlimited opendomain data. Future work includes (1) technical innovations on each component of our system for further performance improvements, (2) exploring self-training on the NLU side too to evolve both the NLU and NLG model at the same time.

\section{Acknowledgments}

We would like to thank the first author of Peng et al. (2020), Baolin Peng, for his generous help. We also thank the anonymous reviewers for their helpful comments.

\section{References}

Tom B Brown, Benjamin Mann, Nick Ryder, Melanie Subbiah, Jared Kaplan, Prafulla Dhariwal, Arvind Neelakantan, Pranav Shyam, Girish Sastry, Amanda Askell, et al. 2020. Language models are few-shot learners. arXiv preprint arXiv:2005.14165.

Paweł Budzianowski, Tsung-Hsien Wen, Bo-Hsiang Tseng, Inigo Casanueva, Stefan Ultes, Osman Ramadan, and Milica Gašić. 2018. Multiwoz-a large-scale multi-domain wizard-of-oz dataset for task-oriented dialogue modelling. arXiv preprint arXiv: 1810.00278 .

Jiaao Chen, Zichao Yang, and Diyi Yang. 2020. Mixtext: Linguistically-informed interpolation of hidden space for semi-supervised text classification. In Proceedings of the 58th Annual Meeting of the Association for Computational Linguistics, pages 2147 2157.

Zihang Dai, Zhilin Yang, Yiming Yang, Jaime G Carbonell, Quoc Le, and Ruslan Salakhutdinov. 2019. Transformer-xl: Attentive language models beyond a fixed-length context. In Proceedings of the 57th Annual Meeting of the Association for Computational Linguistics, pages 2978-2988.
Jacob Devlin, Ming-Wei Chang, Kenton Lee, and Kristina Toutanova. 2019. BERT: Pre-training of deep bidirectional transformers for language understanding. In Proceedings of the 2019 Conference of the North American Chapter of the Association for Computational Linguistics: Human Language Technologies, Volume 1 (Long and Short Papers), pages 4171-4186, Minneapolis, Minnesota. Association for Computational Linguistics.

Jingfei Du, Edouard Grave, Beliz Gunel, Vishrav Chaudhary, Onur Celebi, Michael Auli, Veselin Stoyanov, and Alexis Conneau. 2021. Self-training improves pre-training for natural language understanding. In Proceedings of the 2021 Conference of the North American Chapter of the Association for Computational Linguistics: Human Language Technologies, pages 5408-5418, Online. Association for Computational Linguistics.

Ondřej Dušek and Filip Jurčíček. 2016. Sequence-tosequence generation for spoken dialogue via deep syntax trees and strings. In Proceedings of the 54th Annual Meeting of the Association for Computational Linguistics (Volume 2: Short Papers), pages 45-51, Berlin, Germany. Association for Computational Linguistics.

Sergey Edunov, Alexei Baevski, and Michael Auli. 2019. Pre-trained language model representations for language generation. In Proceedings of the 2019 Conference of the North American Chapter of the Association for Computational Linguistics: Human Language Technologies, Volume 1 (Long and Short Papers), pages 4052-4059.

Sergey Edunov, Myle Ott, Michael Auli, and David Grangier. 2018. Understanding back-translation at scale. In Proceedings of the 2018 Conference on Empirical Methods in Natural Language Processing, pages 489-500.

Jianfeng Gao, Michel Galley, and Lihong Li. 2018. Neural approaches to conversational AI. In Proceedings of the 56th Annual Meeting of the Association for Computational Linguistics: Tutorial Abstracts, pages 2-7, Melbourne, Australia. Association for Computational Linguistics.

Silin Gao, Yichi Zhang, Zhijian Ou, and Zhou Yu. 2020. Paraphrase augmented task-oriented dialog generation. In Proceedings of the 58th Annual Meeting of the Association for Computational Linguistics, pages 639-649.

Hongyu Guo, Yongyi Mao, and Richong Zhang. 2019. Augmenting data with mixup for sentence classification: An empirical study. arXiv preprint arXiv:1905.08941.

Matthew Henderson, Paweł Budzianowski, Iñigo Casanueva, Sam Coope, Daniela Gerz, Girish $\mathrm{Ku}$ mar, Nikola Mrkšić, Georgios Spithourakis, PeiHao Su, Ivan Vulic, and Tsung-Hsien Wen. 2019. 
A repository of conversational datasets. In Proceedings of the Workshop on NLP for Conversational AI. Data available at github.com/PolyAILDN/conversational-datasets.

Zhiting Hu, Bowen Tan, Russ R Salakhutdinov, Tom M Mitchell, and Eric P Xing. 2019. Learning data manipulation for augmentation and weighting. In $\mathrm{Ad}$ vances in Neural Information Processing Systems, volume 32, pages 15764-15775. Curran Associates, Inc.

Xiaoqi Jiao, Yichun Yin, Lifeng Shang, Xin Jiang, Xiao Chen, Linlin Li, Fang Wang, and Qun Liu. 2020. TinyBERT: Distilling BERT for natural language understanding. In Findings of the Association for Computational Linguistics: EMNLP 2020, pages 4163-4174, Online. Association for Computational Linguistics.

Mihir Kale and Abhinav Rastogi. 2020. Template guided text generation for task-oriented dialogue. In Proceedings of the 2020 Conference on Empirical Methods in Natural Language Processing (EMNLP), pages 6505-6520, Online. Association for Computational Linguistics.

Juntao Li, Lisong Qiu, Bo Tang, Dongmin Chen, Dongyan Zhao, and Rui Yan. 2019. Insufficient data can also rock! learning to converse using smaller data with augmentation. In Proceedings of the AAAI Conference on Artificial Intelligence, volume 33, pages 6698-6705.

Andrea Madotto, Zhaojiang Lin, Zhenpeng Zhou, Seungwhan Moon, Paul Crook, Bing Liu, Zhou Yu, Eunjoon Cho, and Zhiguang Wang. 2020. Continual learning in task-oriented dialogue systems. arXiv preprint arXiv:2012.15504.

Baolin Peng, Chenguang Zhu, Chunyuan Li, Xiujun $\mathrm{Li}$, Jinchao Li, Michael Zeng, and Jianfeng Gao. 2020. Few-shot natural language generation for task-oriented dialog. In Findings of the Association for Computational Linguistics: EMNLP 2020, pages 172-182, Online. Association for Computational Linguistics.

Jun Quan and Deyi Xiong. 2019. Effective data augmentation approaches to end-to-end task-oriented dialogue. In 2019 International Conference on Asian Language Processing (IALP), pages 47-52. IEEE.

Alec Radford, Jeffrey Wu, Rewon Child, David Luan, Dario Amodei, and Ilya Sutskever. 2019. Language models are unsupervised multitask learners. OpenAI blog, 1(8):9.

Abhinav Rastogi, Xiaoxue Zang, Srinivas Sunkara, Raghav Gupta, and Pranav Khaitan. 2020. Schemaguided dialogue state tracking task at dstc8. arXiv preprint arXiv:2002.01359.

Rico Sennrich, Barry Haddow, and Alexandra Birch. 2016. Improving neural machine translation models with monolingual data. In Proceedings of the 54th Annual Meeting of the Association for Computational Linguistics (Volume 1: Long Papers), pages 86-96.

Van-Khanh Tran and Minh Le Nguyen. 2017. Natural language generation for spoken dialogue system using rnn encoder-decoder networks. In Proceedings of the 21st Conference on Computational Natural Language Learning (CoNLL 2017), pages 442-451.

Van-Khanh Tran, Minh Le Nguyen, and Satoshi Tojo. 2017. Neural-based natural language generation in dialogue using rnn encoder-decoder with semantic aggregation. In Proceedings of the 18th Annual SIGdial Meeting on Discourse and Dialogue, pages 231240.

Tsung-Hsien Wen, Milica Gašić, Nikola Mrkšić, Lina M. Rojas-Barahona, Pei-Hao Su, David Vandyke, and Steve Young. 2016. Multi-domain neural network language generation for spoken dialogue systems. In Proceedings of the 2016 Conference of the North American Chapter of the Association for Computational Linguistics: Human Language Technologies, pages 120-129, San Diego, California. Association for Computational Linguistics.

Tsung-Hsien Wen, Milica Gašić, Nikola Mrkšić, PeiHao Su, David Vandyke, and Steve Young. 2015. Semantically conditioned LSTM-based natural language generation for spoken dialogue systems. In Proceedings of the 2015 Conference on Empirical Methods in Natural Language Processing, pages 1711-1721, Lisbon, Portugal. Association for Computational Linguistics.

Qizhe Xie, Zihang Dai, Eduard Hovy, Thang Luong, and Quoc Le. 2020. Unsupervised data augmentation for consistency training. Advances in Neural Information Processing Systems, 33.

Zijian Zhao, Su Zhu, and Kai Yu. 2019. Data augmentation with atomic templates for spoken language understanding. In Proceedings of the 2019 Conference on Empirical Methods in Natural Language Processing and the 9th International Joint Conference on Natural Language Processing (EMNLPIJCNLP), pages 3628-3634. 


\section{A The calculation of TF-IDF}

To calculate the TF-IDF score for a n-gram phrase, we take all in-domain texts $\mathbb{X}$ as one document $d$ to calculate its TF (Term Frequency) score, and randomly selected open-domain texts as the set of documents $D$ to calculate the IDF (Inverse Document Frequency) score ${ }^{10}$. Thus, we formulate the TF-IDF score for n-gram phrase $p h_{i}$ as:

$$
\operatorname{TF}-\operatorname{IDF}\left(p h_{i}, d, D\right)=\mathrm{tf}\left(p h_{i}, d\right) \cdot \operatorname{idf}\left(p h_{i}, D\right),
$$

where,

$$
\begin{aligned}
\operatorname{tf}\left(p h_{i}, d\right) & =\log \left(1+\operatorname{freq}\left(p h_{i}, d\right)\right) \\
\operatorname{idf}\left(p h_{i}, D\right) & =\log \left(\frac{|D|}{\left|\left\{p h_{i} \in d\right\}\right|}\right),
\end{aligned}
$$

in which, freq $\left(p h_{i}, d\right)$ denotes the raw count of the phrase $p h_{i}$ appears in the document $d$.

\section{B The structure of the BERT-based NLU annotation}

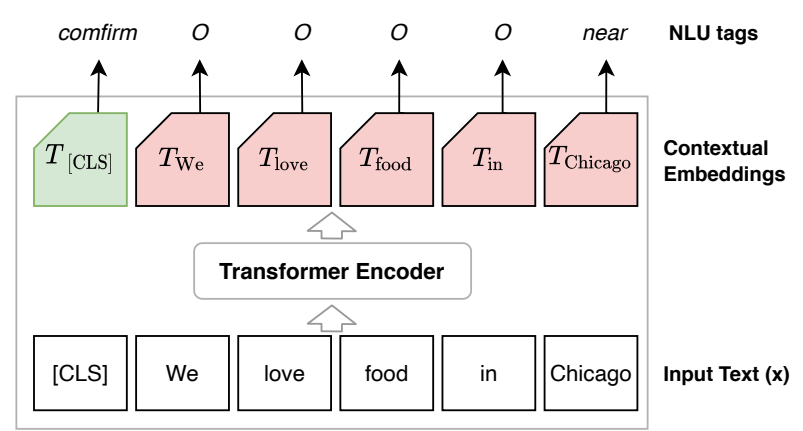

Figure 5: The structure of the BERT-based NLU annotation. The MR for the text "We love food in Chicago" is "confirm ( near = Chicago )". Each slot-value token is annotated with the slot-name. The rest tokens are annotated with " $O$ ".

\section{Statistics for the Augmented Data}

\begin{tabular}{l|ccccccc}
\hline Domains & Restaurant & Laptop & Hotel & TV & Attraction & Train & Taxi \\
\# InD Pairs & 51 & 51 & 51 & 51 & 50 & 50 & 40 \\
\# Keywords (K) & 0.23 & 0.06 & 0.22 & 0.28 & 0.27 & 0.18 & 0.17 \\
\# Rtv Texts (K) & 885.46 & 1000.13 & 760.61 & 850.00 & 1262.69 & 650.53 & 573.93 \\
\# Aug Pairs (K) & 30.97 & 36.62 & 40.46 & 49.76 & 65.48 & 9.60 & 4.95 \\
\# Delex MRs (K) & 0.78 & 5.84 & 0.91 & 6.39 & 0.33 & 0.54 & 0.05 \\
\hline
\end{tabular}

Table 9: FEWSнотWOZ statistics of the augmented pairs over 7 different domains. InD is short for in-domain.

\begin{tabular}{l|cccccccc}
\hline Domains & Restaurants & Hotels & Flights & Calendar & Banks & Weather & Buses & Events \\
\# InD Pairs & 50 & 50 & 50 & 25 & 23 & 11 & 50 & 50 \\
\# Keywords (K) & 0.23 & 0.15 & 0.25 & 0.09 & 0.05 & 0.04 & 0.17 & 0.17 \\
\# Rtv Texts (K) & 1021.64 & 1068.43 & 1195.41 & 582.22 & 112.78 & 387.90 & 749.46 & 1305.41 \\
\# Aug Pairs (K) & 61.51 & 20.64 & 39.59 & 56.87 & 1.27 & 6.39 & 11.15 & 56.55 \\
\# Delex MRs (K) & 1.15 & 0.77 & 1.64 & 0.19 & 0.03 & 0.04 & 1.31 & 1.05 \\
\hline \hline Model & Homes & Media & Movies & Music & Rentalcars & Ridesharing & Services & Travel \\
\# InD Pairs & 21 & 14 & 30 & 21 & 50 & 48 & 50 & 14 \\
\# Keywords (K) & 0.07 & 0.04 & 0.08 & 0.08 & 0.12 & 0.18 & 0.22 & 0.04 \\
\# Rtv Texts (K) & 403.91 & 335.42 & 538.68 & 1033.63 & 469.95 & 1180.02 & 953.51 & 362.45 \\
\# Aug Pairs (K) & 8.04 & 3.90 & 5.90 & 29.69 & 6.41 & 27.02 & 60.09 & 14.80 \\
\# Delex MRs (K) & 0.15 & 0.05 & 0.06 & 0.13 & 0.20 & 0.23 & 2.00 & 0.05 \\
\hline
\end{tabular}

Table 10: FEwSHOTSGD statistics of the augmented pairs over 16 domains. InD is short for in-domain.

\footnotetext{
${ }^{10}$ Here, each open-domain text represents a document.
} 


\begin{tabular}{|c|c|c|c|c|c|c|c|c|}
\hline Nvt. $(\%) \downarrow$ & Data & Restaurant & Laptop & Hotel & TV & Attraction & Train & Taxi \\
\hline Nvt. uni & & 12.46 & 28.93 & 11.36 & 27.55 & 08.84 & 12.19 & 09.22 \\
\hline Nvt. bi & $=$ & 48.70 & 69.82 & 46.68 & 72.53 & 46.66 & 46.13 & 35.40 \\
\hline Nvt. tri & 7 & 77.21 & 88.68 & 74.33 & 91.33 & 75.48 & 70.75 & 62.69 \\
\hline Nvt. four & & 91.07 & 97.02 & 88.46 & 97.94 & 90.49 & 84.74 & 80.46 \\
\hline Nvt. uni & & 11.20 & 06.33 & 06.13 & 04.51 & 04.09 & 09.80 & 07.56 \\
\hline Nvt. bi & ש & 39.45 & 37.86 & 31.37 & 25.72 & 21.60 & 38.68 & 39.00 \\
\hline Nvt. tri & 2 & 73.63 & 69.10 & 66.98 & 61.10 & 53.72 & 80.21 & 79.10 \\
\hline Nvt. four & & 93.73 & 89.63 & 89.10 & 88.39 & 79.92 & 96.28 & 95.87 \\
\hline
\end{tabular}

Table 11: N-gram novelty $(\downarrow)$ breakdowns in FEWSHOTWOZ.

\begin{tabular}{|c|c|c|c|c|c|c|c|c|c|}
\hline Nvt. (\%) $\downarrow$ & Data & Restaurants & Hotels & Flights & Calendar & Banks & Weather & Buses & Events \\
\hline Nvt. uni & $\stackrel{2}{=}$ & 18.41 & 14.16 & 14.23 & 25.41 & 16.06 & 25.60 & 18.11 & 21.51 \\
\hline Nvt. bi & ฮี & 58.08 & 53.84 & 59.32 & 69.45 & 49.91 & 72.73 & 66.16 & 62.22 \\
\hline Nvt. tri & $\theta$ & 79.82 & 74.84 & 84.08 & 86.67 & 72.36 & 88.47 & 87.07 & 85.09 \\
\hline Nvt. four & & 91.62 & 85.00 & 93.75 & 94.49 & 84.25 & 96.43 & 94.14 & 93.10 \\
\hline Nvt. uni & & 02.97 & 03.64 & 01.78 & 02.30 & 08.45 & 04.22 & 02.17 & 02.38 \\
\hline Nvt. bi & 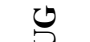 & 18.94 & 21.63 & 19.54 & 17.22 & 37.91 & 34.61 & 28.09 & 19.88 \\
\hline Nvt. tri & \& & 51.72 & 62.72 & 61.20 & 49.51 & 68.73 & 75.77 & 72.05 & 53.82 \\
\hline Nvt. four & & 81.62 & 88.40 & 89.62 & 79.57 & 87.10 & 93.30 & 93.15 & 82.53 \\
\hline Nvt. (\%) $\downarrow$ & Data & Homes & Media & Movies & Music & Rentalcars & Ridesharing & Services & Travel \\
\hline Nvt. uni & $\infty$ & 30.73 & 30.85 & 32.06 & 35.91 & 19.81 & 15.71 & 21.59 & 42.11 \\
\hline Nvt. bi & ב & 77.08 & 73.92 & 73.53 & 77.84 & 57.08 & 51.60 & 60.56 & 83.76 \\
\hline Nvt. tri & 2 & 90.78 & 88.90 & 87.20 & 91.35 & 76.91 & 78.27 & 81.44 & 93.68 \\
\hline Nvt. four & $\Xi$ & 95.22 & 93.68 & 92.77 & 96.55 & 87.09 & 89.74 & 92.37 & 97.59 \\
\hline Nvt. uni & & 08.22 & 10.57 & 08.86 & 03.59 & 01.92 & 03.68 & 03.76 & 06.47 \\
\hline Nvt. bi & ש & 37.85 & 60.10 & 47.53 & 32.38 & 26.68 & 25.48 & 24.82 & 38.29 \\
\hline Nvt. tri & 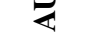 & 75.64 & 93.48 & 81.17 & 73.03 & 68.75 & 68.60 & 55.26 & 73.45 \\
\hline Nvt. four & & 92.74 & 98.75 & 93.12 & 93.37 & 91.65 & 92.50 & 80.79 & 90.90 \\
\hline
\end{tabular}

Table 12: Nvt. $(\downarrow)$ breakdowns in FEwSHOTSGD. EXIST are from the SGD, we compare with in-domain pairs.

\section{Few-shot NLU Performance}

\begin{tabular}{l|ccccccc}
\hline Metrics & Restaurant & Laptop & Hotel & TV & Attraction & Train & Taxi \\
Precision & 1.000 & .8229 & .7500 & .7904 & .6050 & .6552 & .6178 \\
Recall & 1.000 & .8490 & .7500 & .8382 & .6904 & .6706 & .7239 \\
F1 score & 1.000 & .8357 & .7500 & .8136 & .6449 & .6628 & .6667 \\
\hline
\end{tabular}

Table 13: NLU evaluation for FEWSHOTWOZ (Precision $\uparrow)$, (Recall $\uparrow$ ), (F1 score $\uparrow$ )

\begin{tabular}{l|cccccccc}
\hline Metrics & Restaurants & Hotels & Flights & Calendar & Banks & Weather & Buses & Events \\
\hline Precision & .6346 & .6516 & .7229 & .8332 & .8971 & .7177 & .6289 & .5333 \\
Recall & .6635 & .6866 & .7560 & .8897 & .8684 & .7183 & .6372 & .5870 \\
F1 score & .6487 & .6686 & .7391 & .8605 & .8825 & .7180 & .6330 & .5589 \\
\hline \hline Metrics & Homes & Media & Movies & Music & Rentalcars & Ridesharing & Services & Travel \\
Precision & .8201 & .6404 & .4787 & .8011 & .5183 & .6145 & .5470 & .8132 \\
Recall & .8166 & .5060 & .3996 & .7889 & .5288 & .6546 & .6146 & .8713 \\
F1 score & .8183 & .5653 & .4356 & .7950 & .5235 & .6340 & .5788 & .8412 \\
\hline
\end{tabular}

Table 14: NLU evaluation for FEwSHOTSGD (Precision $\uparrow),($ Recall $\uparrow),(F 1$ score $\uparrow)$.

\section{E Generation Examples}

\begin{tabular}{|c|c|}
\hline \multicolumn{2}{|r|}{ Domain: Hotel } \\
\hline $\begin{array}{l}\text { Input MR } \\
\text { Reference } \\
\text { SC-GPT } \\
\text { AUGNLG-SC }\end{array}$ & $\begin{array}{l}\text { inform (name = parker guest house; area = mastro; address }=520 \text { church street; phone }=4156213222 \text { ) } \\
\text { parker guest house is near mastro at } 520 \text { church street. their phone number is } 4156213222 \text {. } \\
\text { the parker guest house is located in mastro and is at } 520 \text { church street. the phone number is } 4156213222 \text {. } \\
\text { the parker guest house in mastro's address is } 520 \text { church street. the phone number is } 4156213222 \text {. }\end{array}$ \\
\hline \multicolumn{2}{|r|}{ Domain: Train } \\
\hline $\begin{array}{l}\text { Input MR } \\
\text { Reference } \\
\text { SC-GPT } \\
\text { AUGNLG-SC }\end{array}$ & $\begin{array}{l}\text { inform (day= sunday; id =tr5413; arriveby=00:04) @ book (none) } \\
\text { train tr5413 arrives at 24:44 or 00:04 on sunday. would you like to book it now? } \\
\text { tr5413 arrives at 00:04. would you like me to book it for you? } \\
\text { tr5413 arrives at 00:04 on sunday. would you like to book? }\end{array}$ \\
\hline \multicolumn{2}{|r|}{ Domain: Taxi } \\
\hline $\begin{array}{l}\text { Input MR } \\
\text { Reference } \\
\text { SC-GPT } \\
\text { AUGNLG-SC }\end{array}$ & $\begin{array}{l}\text { inform (arriveby=16:00) } \\
\text { yes, the taxi is due to arrive at 16:00. } \\
\text { yes, the taxi will arrive by 16:00. } \\
\mathrm{i} \text { ' } \mathrm{m} \text { on it, the taxi will arrive by } 16: 00 \text {. }\end{array}$ \\
\hline
\end{tabular}

Table 15: Generation examples in "Hotel", "Train" and "Taxi" domain on FEWSHOTWOZ. In the same example, the BLEU scores for the SC-GPT generations are higher than the AUGNLG-SC generations. 


\begin{tabular}{|c|c|}
\hline \multicolumn{2}{|r|}{ Domain: Banks } \\
\hline Input MR & $O F F E R($ balance $=2,890.54 ;$ account_type $=$ checking $)$ \\
\hline Reference & your checking balance is $2,890.54$. \\
\hline FT-GPT & now you have $2,890.54$ to spend on goods and services . \\
\hline AUGNLG-FT & balance of checking account is $2,890.54$ \\
\hline \multicolumn{2}{|r|}{ Domain: Buses } \\
\hline Input MR & $O F F E R($ departure $t$ time $=10: 50$ am;price $=42 ;$ fare type $=$ economy $)$ \\
\hline Reference & there is a bus leaving at $10: 50$ am . its economy ticket costs 42 \\
\hline FT-GPT & please confirm that you want to leave from $10: 50$ am to $10: 50 \mathrm{pm}$ \\
\hline AUGNLG-FT & sure, when do you want the bus to leave at? $10: 50 \mathrm{am}$, economy ticket or 42 ? \\
\hline \multicolumn{2}{|r|}{ Domain:Calendar } \\
\hline Input MR & OFFER(available_start_time $=8$ am;available_end_time $=7: 30 \mathrm{pm}) @$ INF_COUNT (count) \\
\hline Reference & yes, you have 2 empty slots from 8 am to $7: 30 \mathrm{pm}$. \\
\hline FT-GPT & you have 1 available seat . \\
\hline AUGNLG-FT & is your event scheduled to start at 8 am and end at 7:30 pm ? \\
\hline \multicolumn{2}{|r|}{ Domain: Events } \\
\hline Input MR & CONFIRM(number_of_tickets $=1 ;$ city $=$ new york $)$ \\
\hline Reference & no problem , i 'll make it 1 ticket for new york. \\
\hline FT-GPT & please confirm your number of tickets. \\
\hline AUGNLG-FT & 1 ticket to the event in new york \\
\hline \multicolumn{2}{|r|}{ Domain: Flights } \\
\hline Input MR & CONFIRM(airlines $=$ southwest airlines; $r$ return_date $=$ march $12 \mathrm{th})$ \\
\hline Reference & so you want a southwest airlines flight that returns on march 12 th ? \\
\hline FT-GPT & it arrives on march 12 th at southwest airlines. \\
\hline AUGNLG-FT & it's not an upgrade, it's a southwest airlines flight that leaves on march 12 th . \\
\hline \multicolumn{2}{|r|}{ Domain: Homes } \\
\hline Input MR & INFORM(phone number =650-961-2975;furnished =false) \\
\hline Reference & the phone number is $650-961-2975$. and the apartment is not furnished . \\
\hline FT-GPT & you are willing to call $650-961-2975$ to schedule an appointment . \\
\hline AUGNLG-FT & $650-971-2975$ is the phone number for the furnished apartment . \\
\hline \multicolumn{2}{|r|}{ Domain: Movies } \\
\hline Input MR & OFFER(movie_name=hackers, high life, sunset) \\
\hline Reference & $\mathrm{i}$ found hackers, high life, and sunset for you \\
\hline FT-GPT & what movie would you like? \\
\hline AUGNLG-FT & what movies are you interested in seeing then ? \\
\hline \multicolumn{2}{|r|}{ Domain: Music } \\
\hline Input MR & CONFIRM(song_name $=$ mile high;playback_device $=$ bedroom speaker $)$ \\
\hline Reference & just to make sure, you want to listen to mile high on the bedroom speaker . \\
\hline FT-GPT & you are listening to the song mile high by mile high . \\
\hline AUGNLG-FT & what is your favorite song from the mile high club? ( or any song for that matter) \\
\hline \multicolumn{2}{|r|}{ Domain: Rentalcars } \\
\hline Input MR & REQUEST(pickup_date = march 5th;dropoff_date $=$ march 9th) \\
\hline Reference & would you like to rent it on march 5 th and return it on march 9 th ? \\
\hline FT-GPT & what date would you like the car to arrive from? \\
\hline AUGNLG-FT & will you be picking it up on march 5th and returning it on march 9th ? \\
\hline \multicolumn{2}{|r|}{ Domain: Ridesharing } \\
\hline Input MR & INFORM(wait_time $=2$; ride_fare $=36.01)$ \\
\hline Reference & the cab will arrive in 2 minutes and it's going to cost 36.01 . \\
\hline FT-GPT & what is the fare for $2 ?$ \\
\hline AUGNLG-FT & please wait 2 hours and 36.01 will be added to your cart . \\
\hline \multicolumn{2}{|r|}{ Domain: Services } \\
\hline Input MR & INFORM(is_unisex=true;phone number=925-446-4144) @ NOTIFY_SUCCESS (none) \\
\hline Reference & it is uniesex and you have an appointment set . phone them at $925-446-4144$. \\
\hline FT-GPT & your appointment has been scheduled the salon is unisex and phone is $925-446-4144$. \\
\hline AUGNLG-FT & your appointment is confirmed . the unisex bathroom is located at $925-446-4144$. \\
\hline \multicolumn{2}{|r|}{ Domain: Weather } \\
\hline Input MR & INFORM(humidity $=43)$ \\
\hline Reference & it will be around $43 \%$. \\
\hline FT-GPT & the humidity will be 43 percent . \\
\hline AUGNLG-FT & the humidity is 43 percent \\
\hline \multicolumn{2}{|r|}{ Domain: Media } \\
\hline Input MR & INFORM(directed_by=roxann dawson) @ NOTIFY_SUCCESS (none) \\
\hline Reference & okay, $\mathrm{i}$ started the movie . the director is roxann dawson. \\
\hline FT-GPT & the movie has started. \\
\hline AUGNLG-FT & it's directed by roxann dawson and it's calledn'tify success . \\
\hline
\end{tabular}

Table 16: Randomly sampled generation examples from FEWSHOTSGD. 